\title{
Prime Numbers: A Particle in a Box and the Complex Wave Model
}

\author{
Raúl Alberto Ribeiro Correia de Sousa
}

Correspondence: Rua Mário de Almeida n.6 2 Esq. Tras. 4700-395 Braga, Portugal.

E-mail: wavetheoryprimenumbers@outlook.com

\author{
Received: September 2, 2015 Accepted: September 15, 2015 Online Published: October 27, 2015 \\ doi:10.5539/jmr.v7n4p43 URL: http://dx.doi.org/10.5539/jmr.v7n4p43
}

\begin{abstract}
Euler's formula establishes the relationship between the trigonometric function and the exponential function. In doing so unifies two waves, a real and an imaginary one, that propagate through the Complex number set, establishing relation between integer numbers. A complex wave, if anchored by zero and by a defined integer number $N$, only can assume certain oscillation modes. The first mode of oscillation corresponds always to a $N$ prime number and the other modes to its multiples.

$$
\psi(x)=x e^{i\left(\frac{n \pi}{N} x\right)}
$$

Under the above described conditions, these waves and their admissible oscillation modes allows for primality testing of integer numbers, the deduction of a new formula $\pi(x)$ for counting prime numbers and the identification of patterns in the prime numbers distribution with computing time gains in the calculations. In this article, four theorems and one rule of factorizing are put forward with consequences for prime number signaling, counting and distribution. Furthermore, it is establish the relationship between this complex wave with a time independent semi-classical harmonic oscillator, in which the spectrum of the allowed energy levels are always only prime numbers. Thus, it is affirmative the reply to the question if the prime numbers distribution is related to the energy levels of a physical system.
\end{abstract}

Keywords: prime numbers, prime wave model, primality, prime number distribution, energy levels, energy spectrum, semi-classical harmonic oscillator, hermitian operator, riemann zeta

AMS subject classifications 11A41, 11A51, 11M26, 35P99, 35Q15, 35Q40, 81S99, $81 \mathrm{~V} 99$

\section{Introduction}

Although for Mathematics, numbers are dimensionless and appear to be abstract, it seems that there are some rules guiding the construction of these abstractions. Prime Numbers (PNs) are generally considered as the building blocks of the Integer set, since that PNs are the only integer numbers that can not be obtain by the multiplication of two other integer numbers (Crandall and Pomerance, 2010). Several formulas are know to calculate PNs. Wilsons theorem is a good example of one of these formulas (Dalawat, 2007). The overwhelming majority of these formulas is considered useless from the practical point of view, each with more or less theoretical interest. Some of these formulas also allow counting primes or help establish limits for the $\pi(x)$ function. Similarly, we also seek to recognize and explain the existence of patterns in PNs, albeit with considerable limitation. Recently, some authors have made progress in establishing the maximum distance between consecutive PNs, using a combination of mathematical techniques (Zhang, 2014; Ford, Green, Konyagin, and Tao, 2014). But, at this moment, still there is no explanation for their singular properties, neither for their distribution across the Integer set. The Hilbert-Polya conjecture suggest that the non-trivial zeros of the Riemann zeta function can be the spectrum of an hermitian operator (Andrade, 2013). Barry and Keating speculate that the Riemann zeta function dynamics is related with the semiclassical hamiltonian $H_{c l}=x p$ (Berry and Keating, 1999). This approach has been developed by several authors, Sierra-Laguna or Menezes-Svaiter for example, where it is search a physics solution for the PNs and Riemann hypothesis (Sierra, 2010, 2008; Gutzwiller, 1990; Sierra and Rodriguez-Laguna, 2011). This is discussed further by Daniel Schumayer and David Hutchinson, on a review and analysis of the physical extension of the Riemann hypothesis(Schumayer and Hutchinson, 2011). And considering Volochis suggestion that the numbers can be the fundamental entities that obey the fundamental laws invariant to the field, as can be seen in the transformation of real numbers into complex numbers often used to solve problems in physics, the author will undertake an approach based on the assumption that numbers are a manifestation of a complex standing wave, oscillating under certain conditions (Volovich, 2010).

In quantum mechanics, the admissible eigenvalues for an atomic electron are related to the constrains imposed by the potential well where it is allowed to exist. Following a similar line of thought to the one used on quantum mechanics, numbers can be regarded as a consequence of allowed oscillation modes, or wavelengths, under certain constrains, similar 
to the potential well in quantum mechanics.

Considering a complex wave represented in eq. 1, as the one defined by Euler's formula, from $-\infty$ to $+\infty$, composed by two different waves oscillating in perpendicular planes to themselves and to the direction of the movement. The cosine wave is oscillating in a plane that is the Real numbers set and the sine wave in the Imaginary numbers set plane, as generally illustrated in Figure 1 (Euler, 2001).

$$
e^{i \theta}=\cos \theta+i \sin \theta
$$

As a consequence, this complex wave will vary its value from only real value, through all complex intermediate values to only imaginary value. In doing so, it establishes a relation between its specific solutions depending on pre-conditions.

In the following sections, the pre-conditions established and its consequences are discussed.

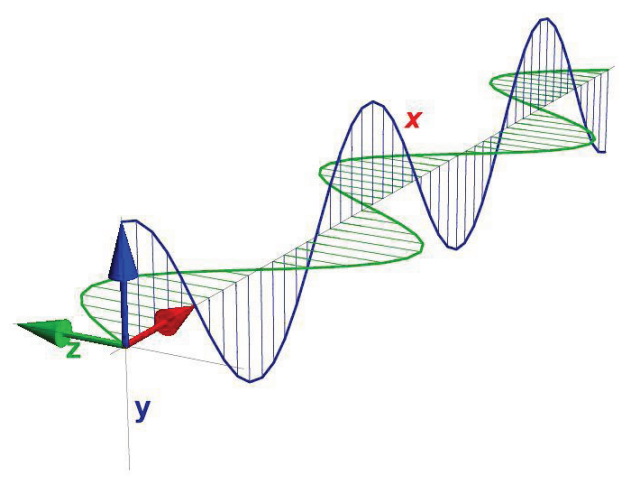

Figure 1. $f(\theta)=e^{i \theta}=\cos \theta+i \sin \theta$ represent over a real (XY plane coloured blue) and an imaginary plane (XZ plane coloured green).

\section{Discussion}

Let us consider a string from $-\infty$ to $+\infty$, represented by the waveform of an imaginary number. As any string anchored to two points, if it is allowed to vibrate freely, only some modes of vibration, or standing wave, are possible. So, the border and initial conditions are such that:

- the string is always anchored at two points, $x=0$ and $x=\mathrm{N}$, an integer number:

$$
\psi(0)=0, \psi(N)=0
$$

- the wave is harmonic and stationary in time, with amplitude depending on $x$ :

$$
\psi(x)=x \sin x
$$

Considering Euler's formula in eq. (1), we can infer that this wave, constrained to the Imaginary numbers set by the sine oscillation, has an associated oscillation in the Real numbers set represented by the cosine term. So, this imaginary oscillation is nothing more than a manifestation of the complex wave in the Complex set. Therefore:

$$
\psi(x)=x[\cos x+i \sin x]
$$

From the general equation of harmonic standing wave, we have that:

$$
\psi(x)=x[\cos (k x)+i \sin (k x)]
$$

where $k$ is defined as eq. (6) by the definition of the harmonic standing wave. 


$$
k=\frac{2 \pi}{\lambda_{n}}
$$

Observing the border conditions of the oscillation in a string with the endings anchored to zero and $N$, the wavelength modes allowed are defined as:

$$
N=n \frac{\lambda_{n}}{2}, n=1,2,3, \ldots
$$

Replacing eq. (6) and eq. (7) in eq. (5),

$$
\psi(x, n, N)=x\left[\cos \left(\frac{n \pi}{N} x\right)+i \sin \left(\frac{n \pi}{N} x\right)\right], n=1,2,3, \ldots
$$

Or, in exponential form,

$$
\psi(x, n, N)=x e^{i\left(\frac{n \pi}{N} x\right)}, n=1,2,3, \ldots
$$

So, $\psi(x)$ is a complex harmonic standing wave in a string constrained by an integer number $N$, with oscillation mode $n$ allowed according to the eq. (8) or (9). This wave, despite being anchored to zero and $N$, propagates to $-\infty$ to $+\infty$ in a constant frequency dependent on $N$ and $n$, with amplitude dependent on $x$. For a given $N$ and $n$, let us represent $\psi(x)$ as the $N^{n} W$ (ave). The oscillation mode $n=1$ is represented as the fundamental oscillation mode or $N^{1} W$.

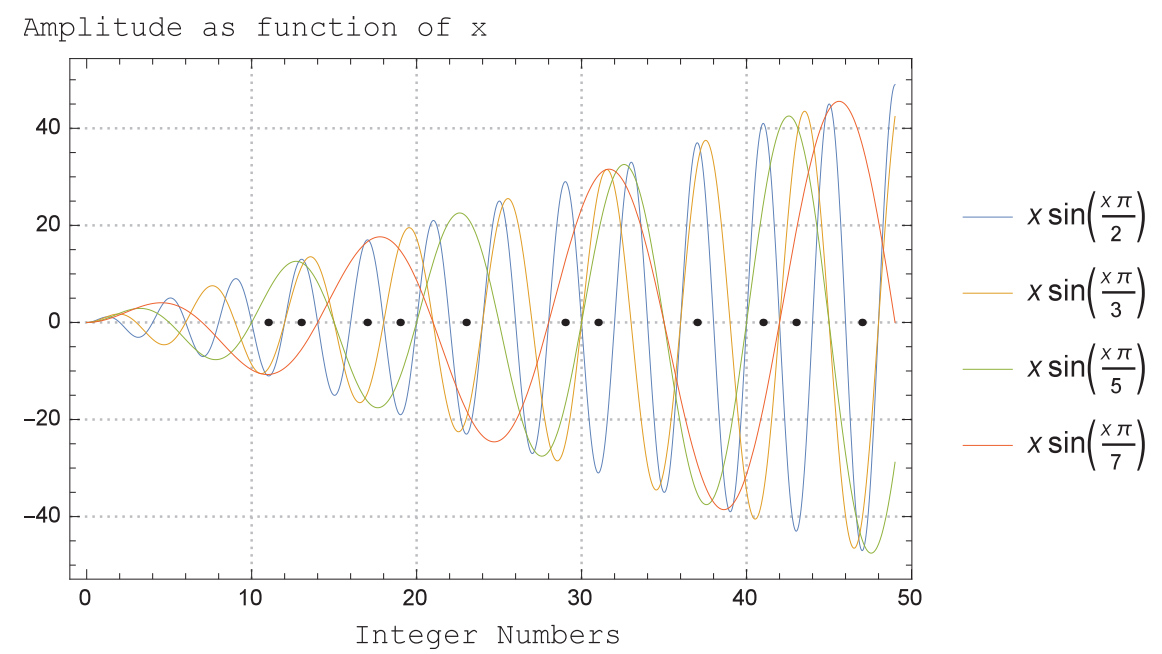

Figure 2. $N^{1} W$ (Fundamental oscillation mode for $\operatorname{Im}(\psi(x))$ for given $N$ ) for $N=\{2,3,5,7\}$ with gaps for $\{11,13,17,19,23,29,31,37,41,43,47\}$.

This wave will always have complex value, except when the $\sin \left(\frac{n \pi}{N} x\right)$ becomes zero. In that circumstance, $\psi(x)$ will have non-imaginary value or, in other words, a pure real number because $\cos \left(\frac{n \pi}{N} x\right)$ becomes equal to one, since $\operatorname{Im}[\psi(x)]=0$ and $\operatorname{Re}[\psi(x)] \neq 0$.

As can be seen from fig. 2, if we represent the $N^{1} W$ for the first four PNs, $\mathrm{N}=\{2,3,5,7\}$, the $\psi(x)$ waves leave some gaps when crossing the $x$-axis in several integers. These gaps signal only and all PNs between $N=7$ and $N^{2}=49$. In fig. 2 , the $\operatorname{Im}(\psi(x))$ is represented with its true amplitude dependence on $x$. The same figure can be made for the $\operatorname{Re}(\psi(x))$ but with $\frac{\pi}{2}$ phase delay due to the cosine. 


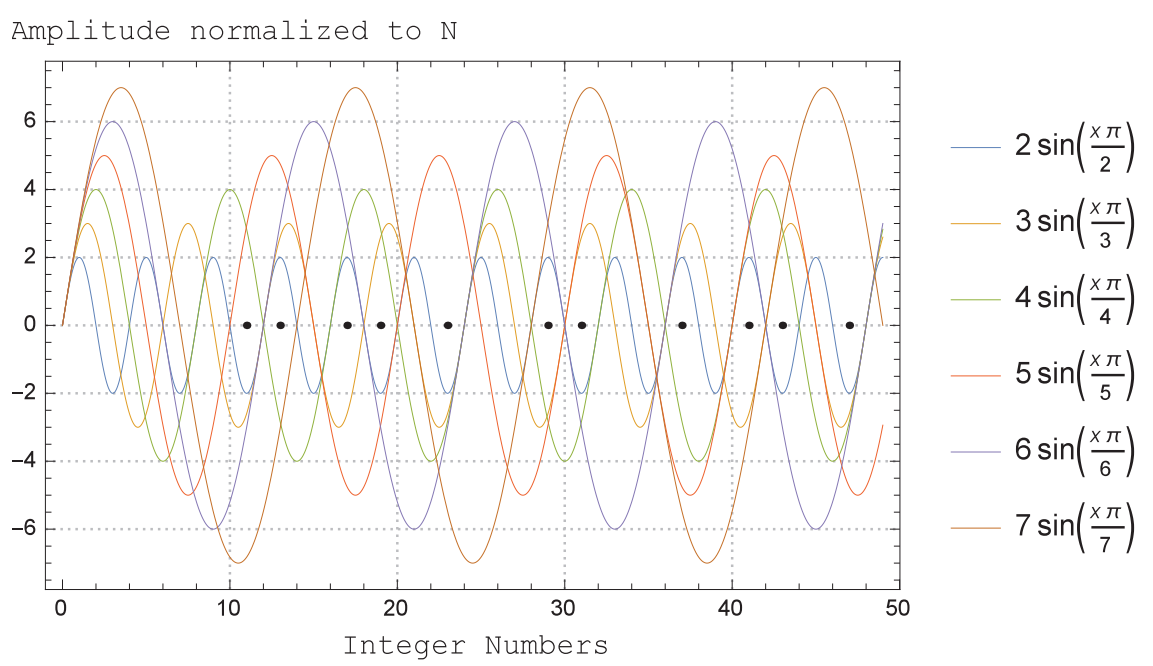

Figure 3. $N^{1} W$ (Fundamental oscillation mode for $\operatorname{Im}(N \psi(x) / x)$ for given $\mathrm{N}$ ) for $N=\{2,3,4,5,6,7\}$ with gaps for $\{11,13,17,19,23,29,31,37,41,43,47\}$.

Fig. 3 represents each wave $\operatorname{Im}(N \psi(x) / x)$ with the amplitude equals to $N$, with the purpose of clearly highlighting these PNs gaps. Also, in fig. 3 , all $N^{1} W$ up to $\mathrm{N}=7$, that is $\mathrm{N}=\{2,3,4,5,6,7\}$, are represented highlighting that, when searching for PNs, there is no difference between using all $N^{1} W$ up to a certain integer number or all $\mathrm{N}$ that are PNs up to that certain integer number.

\subsection{Signalizing Prime Numbers}

The behavior of the complex wave described above may be used to signal PNs. The following theorems enunciate how to do it.

Theorem 1. For all $x, n$ and $N$ integers excluding zero and $x \leq n<N, \operatorname{Im}[\psi(x)]=0$ does not have solution for all and only $N$ prime number.

$$
\forall x, n \in \mathbb{Z} /\{0\} \wedge \forall N \in P N s \wedge x \leq n<N:\{\operatorname{Im}[\psi(x, n, N)]=0\}=\emptyset
$$

Proof. The same is:

$$
\forall x, n \in \mathbb{Z} /\{0\} \wedge \forall N \in \mathrm{PNs} \wedge x \leq n<N:\left\{x \sin \left(\frac{n \pi}{N} x\right)=0\right\}=\emptyset
$$

From eq. (9),

$$
x=0 \wedge \sin \left(\frac{n \pi}{N} x\right)=0
$$

$x$ can not be zero so,

$$
\begin{gathered}
\frac{n \pi}{N} x=0+k \pi, k=1,2,3, \ldots \\
x=k \frac{N}{n}
\end{gathered}
$$

Since $x<N$, it follows that $k<n$. And, because $N$ PN can only be divided integerly by itself and one, $x$ will always be different from an integer. Therefore, $x$ has no solution for eq. (10) for $N$ PN. However, if $N$ is not a PN then there is always a factor of $N$ that solves eq. (14).

This theorem 1 enables us to determine if a given $N$ is PN by determining if there is not any other oscillation mode different from $n=1$ that produces $N$. Basically, if $\sin \left(\frac{n \pi}{N} x\right)$ does not have one oscillation mode $n$ that "links" $x$ and $N$, meaning that $N$ is not a multiple of $x$, then $N$ is PN. The contrary is also true, if $\sin \left(\frac{n \pi}{N} x\right)$ has one oscillation mode $n$ that "links" $x$ and $N$, then $N$ is not PN because it is a multiple of $x$. For example, the oscillation mode $n=2$ of $N=4$ will produce 
$x=2$ solution set for eq. (14), defining the number 4 as not a PN. Another example is $N=6$, represented in figure 4 , that has a wave with a zero solution for $x=3$ in the second oscillation mode $(n=2)$, represented as $6^{2} \widehat{W}$, or $x=2$ in the third oscillation mode, $n=3\left(6^{3} \widehat{W}\right)$, so the full set of solutions for eq. (14) for $N=6$ is $x=\{2,3\}$ that is represented as $6^{2,3} W$.

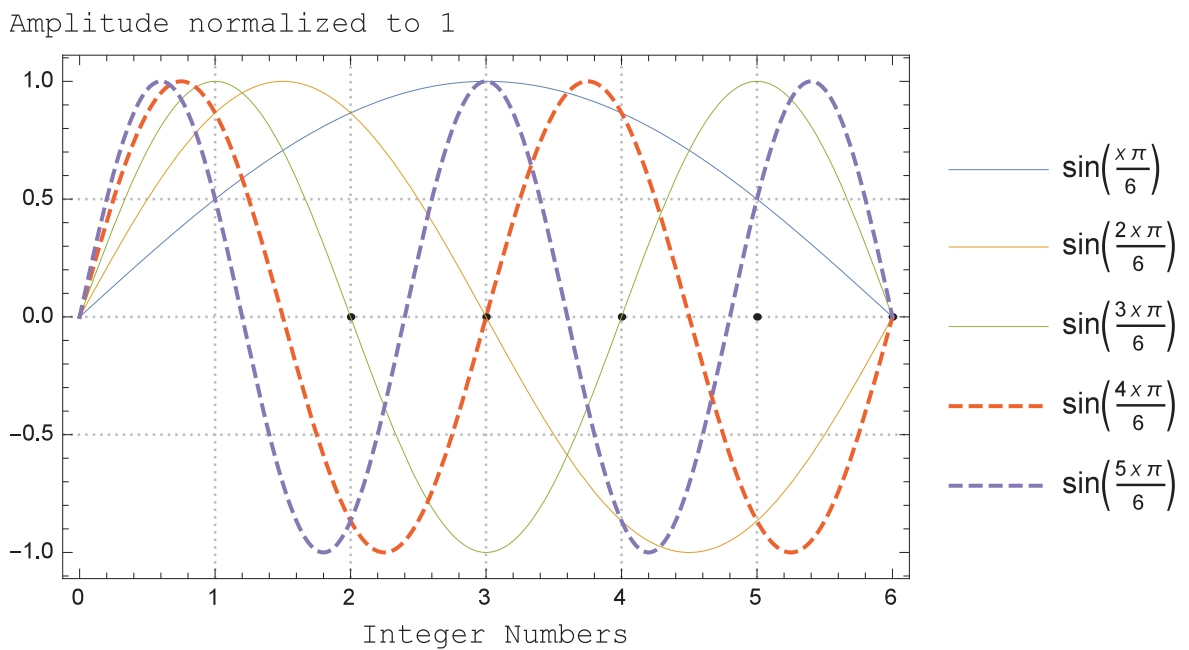

Figure 4. Oscillation modes $n=\{1,2,3,4,5\}$ for $\operatorname{Im}(\psi(x))$ for $N=6$. The possible oscillation modes are represented in a solid line, the others in a dashed line.

But there is no other wave to produce $N=7$ except for the $7^{1} \mathrm{~W}$ as represented in fig 5 . Considering that theorem 1 does not allow $x=\mathrm{N}$, it implies that $n=1$ is always excluded from the solution set. These $N$ prime waves $(P W)$ will be designated as $N P W$, so that $7^{1} W \Leftrightarrow 7 P W$.

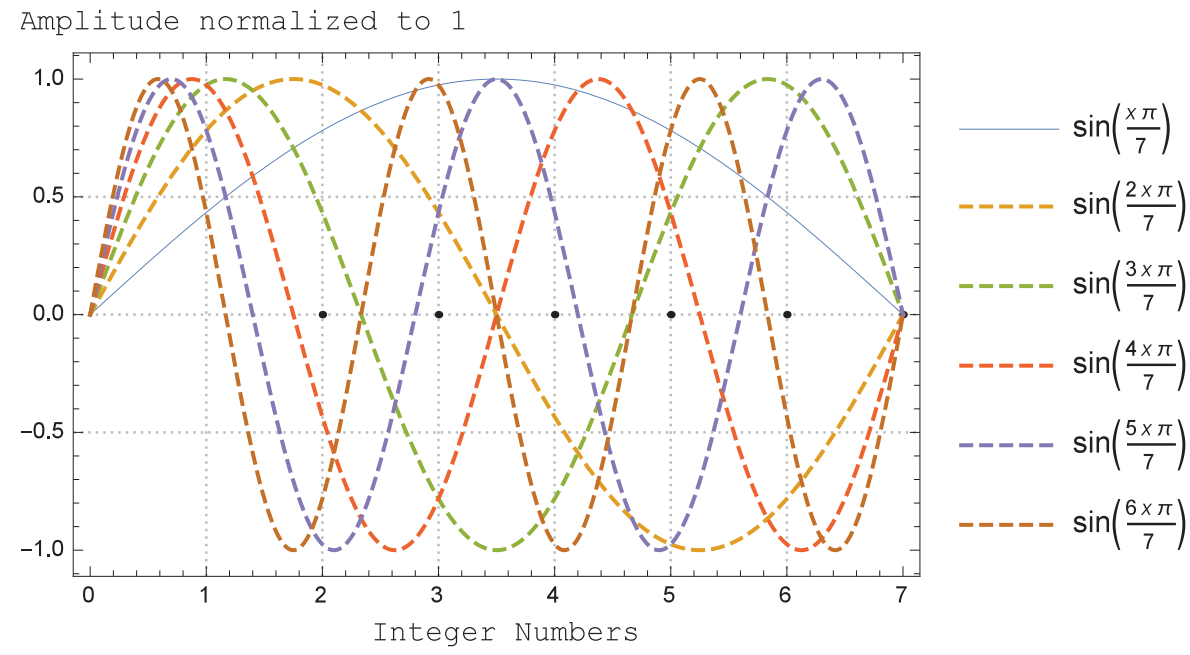

Figure 5. Oscillation modes $n=\{1,2,3,4,5,6\}$ for $\operatorname{Im}(\psi(x))$ for $N=7$. The possible oscillation modes are represented in a solid line, the others in a dashed line.

\subsubsection{Nomenclature}

The widehat $\widehat{W}$ in the $W$, signals that only partial oscillation modes are represented. The absence of the widehat signals that all possible oscillation modes for eq. (14) are represented. From these examples, it is easy to infer that the set of solutions to the theorem 1 , for a given $\mathrm{N}$, is represented by factor $\mathrm{N}$. 


\subsubsection{Primality Test}

For the purpose of primality testing, it should be noted that this generalized form of the theorem 1 can be reduced. In fact, it is only necessary to test all oscillation modes that are PN. All of the other $n$, not PN, are multiples of the $n$ PN and will only produce repeated solutions. So, theorem 1 can be written in the reduce form:

$$
\forall x \in \mathbb{Z} /\{0\} \wedge \forall n, N \in \text { PNs } \wedge x \leq n<N:\left\{x \sin \left(\frac{n \pi}{N} x\right)=0\right\}=\emptyset .
$$

As stated before, PNs are characterized for being the only numbers that can be represented by a $P W$. So, if we consider all existing $P W$ up to a given $N$, we can mix them and build a "merge" function capable of identifying the PNs between $N$ and $N^{2}$. The lower and upper bound can be recursively increased by adding all the PNs, and their $P W \mathrm{~s}$, found in the ]$N, N^{2}$. The process works like a sieve, where each $P W$ is used to exclude every number that is a multiple of the $N$ PN defined by the $P W$ used. The following section addresses the mathematical formulation for the "merge" function.

According to theorem 1, if one wave exists that has an imaginary part zero for one specific $N$ integer number, then this number it is not a PN; consequently, we can create a function, $\alpha(x)$, with the imaginary part of all waves for each integer number from 2 to $N$, in the first oscillation mode $(n=1)$, in such a way that if there exists any zero for this function $\alpha(x)$, for any $x$ greater than $N$, it will signal $x$ as not a PN.

Theorem 2. For all $x, n, m$ and $N$ integers excluding zero and $N<x \leqslant N^{2}$,

$$
\alpha(x): \prod_{m=2}^{N} \operatorname{Im}[\psi(x, n, m)] \neq 0, \text { only has solution for } x \in \text { prime numbers }
$$

Considering that $|\sin (n \pi x)|=\sin (\pi x)$ for $n \in$ integers $^{\dagger}$, the $\alpha(x)$ can be written as:

$$
\alpha(x): \prod_{m=2}^{N} x \sin \left(\frac{\pi}{m} x\right) \neq 0 \text {, only has solution for } x \in \text { prime numbers. }
$$

Since $x$ is always different from zero by theorem imposition, we have that:

$$
\alpha(x): \prod_{m=2}^{N} \sin \left(\frac{\pi}{m} x\right) \neq 0 \text {, only has solution for } x \in \text { prime numbers. }
$$

Proof. Let us consider the product of all existing $N^{1} W$ from $m=2$ to $N$. If there exists a $N^{1} W$ for a given $x$ that has zero imaginary part then, according to theorem 1, $x$ it is not a PN. Therefore, the product of all waves from 2 to $N$ for that $x$ not PN will also be zero and will not be zero for $x$ PN.

This theorem 2 looks for PN ahead of $N$ by searching for an $x$ that is PN, while theorem 1, on the other hand, verifies if $N$ is a PN by looking at the waves behind $N$. The Sieve of Eratosthenes is a consequence of this theorem 2 and therefore is constrained to the same limitations. All $P W$ less than a $N$, that is before a $N$, are used to build a function through the product of the sines, that will have a zero for every $x$ integer that is not a PN. It reflects the way those $P W$ s propagate through the Integer set, linking related integers and leaving gaps on unrelated integers that only can be filled with new totally different $P W \mathrm{~s}$. Due to that particular way of how this function was build, it can only show where all PNs are within a range of $N$ to $N^{2}$. From $N^{2}$ forward, $\alpha(x)$ loses its absolute certainty signalization in finding PNs but never misses identifying one that is not a PN. So that point forward, some of the gaps will be PN and others will be almost PN. Due to $\alpha(x)$ not including all of $P W \mathrm{~s}$ that exist, it is not possible to exclude the almost PNs that factor by a PN that we have not included in $\alpha(x)$, hence the $N^{2}$ limit.

The $\alpha(x)$ explains the apparently random distribution of the PNs and their progressive rareness for an increasing $x$. The first $P W N=\{2,3,5,7\}$, for example, are responsible for the majority of the elimination of integers from the PNs set and, as such, are responsible for the definition of the underlying structure of the distribution of PNs. The other $P W \mathrm{~s}$ greater than 7 will be responsible for the apparent randomness in PN distribution.

As with theorem 1, theorem 2 is also a generalization. Since that for each $N^{n} W$ there exists a $P W$ that behaves the same way, eq. (16) initial proposition can be reduced from "all integers between $m=2$ to $N$ " to "all PNs that exist up to $N$ ". So, the initial condition can be altered to: 
Considering that $|\sin (n \pi x)|=\sin (\pi x) . \dagger$ For all $x$ integers, $n, m$ and $N \in$ Prime Numbers and $N<\mathrm{x} \leqslant N^{2} \ldots$

This is extremely important for computational purposes since it dismisses a lot of unnecessary computations.

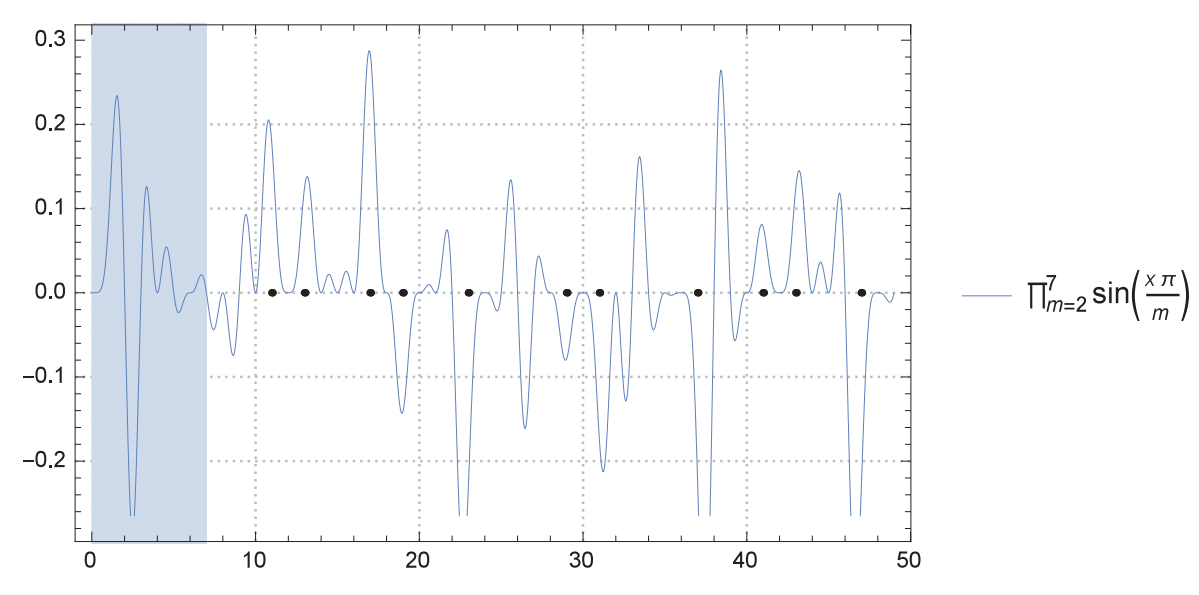

Figure 6. $\alpha(x)=\prod_{m=2}^{7} \sin \left(\frac{\pi}{m} x\right) \wedge m \in$ Prime Numbers.

In fig. 6, it can be seen an example of theorem 2 application with $\alpha(x)$ built with $P W$ from 2 to 7 . All integers $x$ that are not zero of $\alpha(x)$ between 8 and 49 are PN, obeying the limitations stated in theorem 2 .

\subsubsection{Pattern Identification in Prime Numbers Distribution}

The $\alpha(x)$ in fig. 6 has a set of solutions with the period $\tau$ of $\tau=210$, meaning that this function has a pattern and that will repeat exactly the same sequence of zeros for $x+\tau$ for any x greater than 7 . This implies that identifies a pattern, dependent on the prime waves used, that finds PNs and almost PNs. Eq. (19) goes without proof in this article, but $\alpha(x)$ has a global period dependent on the $P W$ s considered in the creation of the function. If only $P W \mathrm{~s}$ are used for building $\alpha(x)$, the $\tau$ can be calculated by:

$$
\tau=\prod_{m=2}^{N} m \wedge m=\{\text { all } P N \text { of each } P W \mathrm{~s} \text { in } \alpha(x)\}
$$

If all $N W \mathrm{~s}$ are used instead of only $P W \mathrm{~s}, \tau$ is the product of all $N$ that are PN and the first prime factor of the each $N$ that are not PNs. For example,

$$
\begin{aligned}
\alpha(x) & =\prod_{m=2}^{9} \sin \left(\frac{\pi}{m} x\right) \wedge m=\{2,3,4,5,6,7,8,9\} \Rightarrow \\
& \Rightarrow \tau=\{2 \times 3 \times 2 \times 5 \times 2 \times 7 \times 2 \times 3\}=2^{4} 3^{2} 5^{1} 7^{1}
\end{aligned}
$$

Generalizing:

$$
\tau=p_{1}^{x} p_{2}^{y} \ldots p_{n}^{z} \wedge p=\{\text { all PNs in } \alpha(x)\}
$$

note: $x, y, \ldots, z$ equal to the number of times that each $P N$ is the lowest prime factor of every integer plus one.

If we use in $\alpha(x)$, all PN from 2 up to $N$ then eq. 19 is the primordial of $N$ :

$$
\tau=N \sharp
$$

A periodic function implies that there is a pattern in the PNs distribution, as the sequence of $\alpha(x)$ zeros repeats each $\tau$, and that this pattern propagates through the Integer set. For $N<x \leq N^{2}$, this pattern will signal PNs and $x \geq N^{2}$ will signal PNs candidates. Every integer excluded is a proven non PN. This same result of the existence of periodicity was found in inductive way by $\mathrm{Xu}$ et al (Xu, Zhang, and Zhou, 2014). 
These patterns are nothing more nor less than the demonstration of the existence of k-tuples. Many patterns, and k-tuples by consequence, can conceived for as many functions as the ones that can be defined by any set of numbers from $k$ to $N$, that does not have to be sequential, such that:

$$
\alpha(x)=\prod_{m=k}^{N} \sin \left(\frac{\pi}{m} x\right) \wedge m \in\{k, \ldots, N\}
$$

This property is significant for the purposes of computational efficiency. Primality testing is a time consuming challenge that can be overcome by the above mentioned property. It is possible to construct and store a set, or database, of matrices with the function zeros for each $\alpha(x)_{k, N}$ ranging from $x$ to $x+\tau$. Computationally, these matrices can be used to filter integers candidates to PNs, in regions distant from where $\alpha(x)$ was produced. These candidates then can be recursively tested with $\alpha(x)$ functions produced by increasingly closer PNs by adjusting $k$ and $N$. One main advantage is to allow for region primality testing for PNs and not just one number at a time, preserving memory, through recording of the patterns, over the calculations made for future progression. Another advantage is the reduction of necessary calculations for every integer number tested since that precalculated filters will be used to excluded non PNs based on its position in the Integer set.

For the purposes of demonstration, let us calculate the pattern of zeros and ones, where zero is a non-PN and one is a PN, produced by $\alpha(x)_{2,97}$ :

$$
\left\lceil\left|\alpha(x)_{2,97}\right|\right\rceil=\left\lceil\left|\prod_{m=2}^{97} \sin \left(\frac{\pi}{m} x\right)\right|\right\rceil \wedge m \in\{2, \ldots, 97\}
$$

The pattern of zeros and ones has a length of 2305567963945518424753102147331756070 numbers, starting from 98 (included), where the ones signals PNs up to 9409 (included) and candidates to PNs from there on.

A possible algorithm for finding PNs recursively, using the $\alpha(x)$ periodic property, is shown below:

- Calculate $\left\lceil\left|\alpha(x)_{2, N}\right|\right\rceil$

- Store

$-\mathrm{N}+1$

- Pattern Length $\tau_{0}=\prod_{m=2}^{N} m \wedge p=\{$ all PNs in $\alpha(x)\}$

- Pattern $[0,1,0, \ldots, 0]$

- Use all PNs in $\left.] N, N^{2}\right]$

- ...

- Calculate $\left[\left|\alpha(x)_{N^{2^{i-2}}, N^{i-1}}\right|\right]$

- Store

$-N^{2^{i-2}+1}$

- Pattern Length $\tau_{0}=\prod_{m=N^{2^{i-2}}}^{N^{2^{i-1}}} m \wedge p=\{$ all PNs in $\alpha(x)\}$

- Pattern $[0,1,0, \ldots, 0]$

- Use all PNs in $\left.] N^{2^{i-2}}, N^{2^{i}}\right]$

- ...

The process of "using all prime numbers" involves checking whether a particular position, corresponding to a certain number, it is not excluded from being PN by a previous pattern. This process has the advantage of creating a persistent database location of primes, which can be progressively increased, automatically and recursively.

\subsection{Prime Counting Function}

The theorem 2 lays the foundation for the $\pi(y)$ prime numbers count function. Since $\alpha(x)$ returns zero for any $x$ not-PN and a different value from zero for PN, a ceiling function can be built that adds all of absolute values of $\alpha(x)$, within the conditions of theorem 2. Defining a ceiling for the $\alpha(x)$ means that this new function returns zero for a not-PN and one for PN, making simple the PNs sum in a specified interval. 
Theorem 3. For all $x, y, m$ and $n$ integers excluding zero,

$$
\left.\pi\left(y^{2}\right)-\pi(y)=\sum_{x=y+1}^{y^{2}}|| \prod_{m=2}^{y} \sin \left(\frac{\pi}{m} x\right) \mid\right] \wedge \pi(y)=\#\{p: p \leqslant y \wedge p \in P N\}
$$

Theorem 3 counts PNs in the interval $\left.] y, y^{2}\right]$ assuming that $\pi(x)$ is previously known. The condition that imposes $m$ as any integer number, is a generalization and can be reduced to "for all $x, y$ and $n$ integers excluding zero, for all $m$ prime numbers...".

\subsection{The Probability of a Prime Candidate being a Prime Number}

For counting PNs, we have used a deterministic approach to find PNs. But the theorem 3 allows the possibility of using an incomplete $\alpha(x)$ function with computational benefits for quick selecting PN candidates for primality testing or deterministic evaluation.

Now we focus on a generic region of the Integer set between $y$ and $y^{2}$ for a comparison between a complete $\alpha(x)$ function that "uses all PNs lesser than $y .$. " and an incomplete $\alpha(x)$ function that "uses all PNs lesser than $l$, such that $l$ is lesser than $y . . . "$.

In this section, we only will use $\alpha(x)$ in the more restricted formulation where $n \in$ PNs, in spite of the more general formulation where $n \in \mathbb{N}$, without any prejudice for the analysis or conclusions.

The theorem 2 (eq. (16)) states that $\alpha(x) \neq 0$ verifies only when for all $x \in \mathrm{PN}$ and $N<x \leq N^{2}$. And, also states that for $x>N^{2}, \alpha(x)$ will signal all PNs and will also signal the almost PNs too, which have prime factors in the region $x>N$. The explanation is simple, if the waves that exclude numbers from being PNs in $N<x \leq N^{2}$ are not included in $\alpha(x)$ then it will be unable to signal them as belonging to one of those un-included waves. Thus, the incomplete $\alpha(x)$ will consider them without any "maker" wave and therefore, looking like a PN. Whenever we refer to $\alpha(x)$ in the $N<x \leq N^{2}$ such that all $P W$ from 2 to $N$ were used in the building of $\alpha(x)$, it will be called the complete $\alpha(x)$. For $x>N^{2}$, we will call it the incomplete $\alpha(x)$ and identify it as $\breve{\alpha_{N}}(x)$. Here follows an example where $\alpha(x)$ is complete for $N<x \leq N^{2} \wedge N=5$ :

$$
\begin{aligned}
& \left.\left.\alpha(x)=\prod_{m=2}^{N} \sin \left(\frac{\pi}{m} x\right) \neq 0 \wedge n \in \mathrm{PNs}, \forall x \text { integer } \in\right] N, N^{2}\right] \\
& \left.\left.\alpha(x)=\prod_{m=2}^{5} \sin \left(\frac{\pi}{m} x\right) \neq 0 \wedge n \in \mathrm{PNs}, \forall x \text { integer } \in\right] 5,5^{2}\right]
\end{aligned}
$$

The solution set for eq. 26 is $x=\{7,11,13,17,19,23\}$. All of these numbers are PNs and also, more importantly, these numbers are all the PNs in the $N<x \leq N^{2}$ region.

Observing the conditions imposed on $\alpha(x)$, all $x$ that satisfies eq. (25) will be PNs according to theorem 2 . Let us look at what happens in the $x>N^{2}$ region. For demonstration purposes, we will restrict the analysis to the $N^{2}<x \leq(2 N)^{2}$ region.

$$
\left.\left.\breve{\alpha}(x)=\prod_{m=2}^{5} \sin \left(\frac{\pi}{m} x\right) \neq 0 \wedge m \in \text { PNs, } \forall x \text { integer } \in\right] 5^{2},(2 \times 5)^{2}\right]
$$

The solution set is $x=\{29,31,37,41,43,47,49,53,59,61,67,71,73,77,79,83,89,91,97\}$. All of these numbers are PNs with the exception for the subset of the almost PN $\{49,77,91\}$. Analyzing each prime factor for these almost PNs, we obtain: $49=\{\{7,2\}\}, 77=\{\{7,1\},\{11,1\}\}$ and $91=\{\{7,1\},\{13,1\}\}$. It is evident that $\breve{\alpha_{5}}(x)$ fails to exclude numbers that have prime factors represented by waves not included in itself, $P W \mathrm{~s}>5 P W$, in this case $7 P W, 11 P W$ and $13 P W$. But it never excludes a number that is a PN. In clinical language, $\breve{\alpha_{N}}(x)$ for $x>N^{2}$ gives some false positives, but no false negatives.

In theorem 3 section, we stated that the probability of any solution of $\alpha_{N}(x)$ being a PN could be determined. The solution is quite simple when observed eq. (24).

$$
\pi\left(y^{2}\right)-\pi(y)=\sum_{x=y+1}^{y^{2}}\left[\left|\prod_{m=2}^{y} \sin \left(\frac{\pi}{m} x\right)\right|\right\rceil \pi(y)=\#\{p: p \leqslant y \wedge p \in \mathrm{PNs}\}
$$


Eq. (24) counts the number of prime numbers that $\alpha(x)$, or $\alpha_{y}(x)$, signals in the $\left.] y, y^{2}\right]$, matching exactly with the total number of primes in the same interval.

For the purpose of clarifying our line of thought, let us substitute $\alpha_{y}(x)$ using the nomenclature for the waves defined in the theorem 1 section.

$$
\pi\left(y^{2}\right)-\pi(y)=\sum_{x=y+1}^{y^{2}}\left[\left|\prod_{m=2}^{y} m^{1} W\right| \mid \wedge \pi(y)=\#\{p: p \leqslant y \wedge p \in \mathrm{PNs}\}\right.
$$

or

$$
\pi\left(y^{2}\right)-\pi(y)=\sum_{x=y+1}^{y^{2}}\left[\left|\alpha_{y}(x)\right|\right] \wedge \pi(y)=\#\{p: p \leqslant y \wedge p \in \mathrm{PNs}\}
$$

The number of candidates to $\mathrm{PN}$ in $\left.] y, y^{2}\right]$ produced by $\breve{\alpha_{l}}(x)$ for any $l<y$ is:

$$
\pi\left(y^{2}\right)-\pi(y)=\sum_{x=y+1}^{y^{2}}\left[\left|\prod_{m=2}^{l} m^{1} W\right| \| \wedge \pi(y)=\#\{p: p \leqslant y \wedge p \in \mathrm{PNs}\}\right.
$$

or

$$
\pi\left(y^{2}\right)-\pi(y)=\sum_{x=y+1}^{y^{2}}\left\lceil\left|\breve{\alpha}_{l}(x)\right|\right\rceil \wedge \pi(y)=\#\{p: p \leqslant y \wedge p \in \mathrm{PNs}\} .
$$

The classical definition of Probability according to Laplace is:

"The probability of an event is the ratio of the number of cases favorable to it, to the number of all cases possible when nothing leads us to expect that any one of these cases should occur more than any other, which renders them, for us, equally possible." (Jaynes and Rosenkrantz, 1989).

Therefore:

$$
\begin{gathered}
P_{\left.\left(1 y, y^{2}\right]\right)}=P_{\left.\left.(\text {of being PN among candidates in }] y, y^{2}\right]\right)} \\
P_{\left.\left(1 y, y^{2}\right]\right)}=\frac{\text { Number of PNs in } \left.] y, y^{2}\right]}{\text { Number of Candidates to PNs in } \left.] y, y^{2}\right]}
\end{gathered}
$$

and

$$
\begin{gathered}
P_{\left.\left(1 y, y^{2}\right]\right)}=\frac{\sum_{x=y+1}^{y^{2}}\left\lceil\left|\alpha_{y}(x)\right|\right]}{\sum_{x=y+1}^{y^{2}}\left\lceil\left|\breve{\alpha}_{l}(x)\right|\right\rceil} \\
P_{\left.\left(1 y, y^{2}\right]\right)}=\frac{\sum_{x=y+1}^{y^{2}}\left[\left|\prod_{m=2}^{y} \sin \left(\frac{\pi}{m} x\right)\right| \mid\right.}{\sum_{x=y+1}^{y^{2}}\left[\left|\prod_{m=2}^{l} \sin \left(\frac{\pi}{m} x\right)\right| \mid\right.}
\end{gathered}
$$

The latter formulation makes apparent the term that influences the probability:

$$
P_{\left.\left(1 y, y^{2}\right]\right)}=\frac{\sum_{x=y+1}^{y^{2}}\left[\left|\prod_{m=l+1}^{y} \sin \left(\frac{\pi}{m} x\right) \cdot \prod_{m=2}^{l} \sin \left(\frac{\pi}{m} x\right)\right|\right]}{\sum_{x=y+1}^{y^{2}}\left[\left|\prod_{m=2}^{l} \sin \left(\frac{\pi}{m} x\right)\right| \mid\right.}
$$

It seems counter-intuitive to evaluate the probability of a candidate being PN and simultaneously to determine exactly how many PNs exist in the same interval. However, eq. (36) provides information on the maximum expected probability in a region where calculations could be possible to execute. This could be useful in some situations, for example, it sets a 
reasonable maximum limit for the probability value in the proximal regions where these calculations can not be executed. Since as we part from zero and we add more $P W$ s to $\alpha(x)$, new PNs become rarer. So, it is possible to build $\breve{\alpha}(x)$ with sufficient $P W \mathrm{~s}$ to achieve a predetermined probability of guessing correctly PNs in the $x>N^{2}$ region. Thus, in the same way, it is possible to calculate the probability of a select integer being a PN for $x>N^{2}$ and, therefore, produce a restricted selection of candidates for possible PNs.

\subsection{About Theorem 1}

Theorem 1 can be written in another useful form.

Theorem 1. For all $x, n$ and $N$ integers excluding zero and $x \leq n<N, \operatorname{Re}[\psi(x)]=1$ does not have solution for all and only $N$ prime number.

$$
\left\{x \cos ^{2}\left(\frac{n \pi}{N} x\right)=1\right\}=\emptyset \text { for } N \in \text { Prime Numbers }{ }^{\ddagger}
$$

Proof. The proof is identical to the one for theorem 1 in page 46 where, the square of the cosine is replaced by the cosine absolute value. However, another simple proof is using the fundamental formula of trigonometry:

$$
\cos ^{2} \theta+\sin ^{2} \theta=1
$$

A simple substitution of the sine shows that, if the sine can not be zero, then the cosine can not be equal to 1 .

\subsection{Factoring Prime Numbers using Prime Waves}

Rule 1. Rule of the fastest and lowest decay

Each $N$ integer factors by the first $n$ that solves,

$$
\sin \left(\frac{n \pi}{N} x\right)=0, n \in \mathbb{N} \wedge x, N \in \mathbb{Z} /\{0,1\}
$$

Each $N$ is divided by the first $n$ that solves eq. (39) with $x$ integer. Then, the factoring continues replacing $N$ by the lowest $x$ that solves eq. (39), until there are no solutions. The resultant set of $n$ and the remainder integer $x$ are factors of $N$.

Any number $N$ will try to decay to the lowest integer number possible as fast as it can. For that, it will always choose the first oscillating mode $n$ that solves eq. (39).

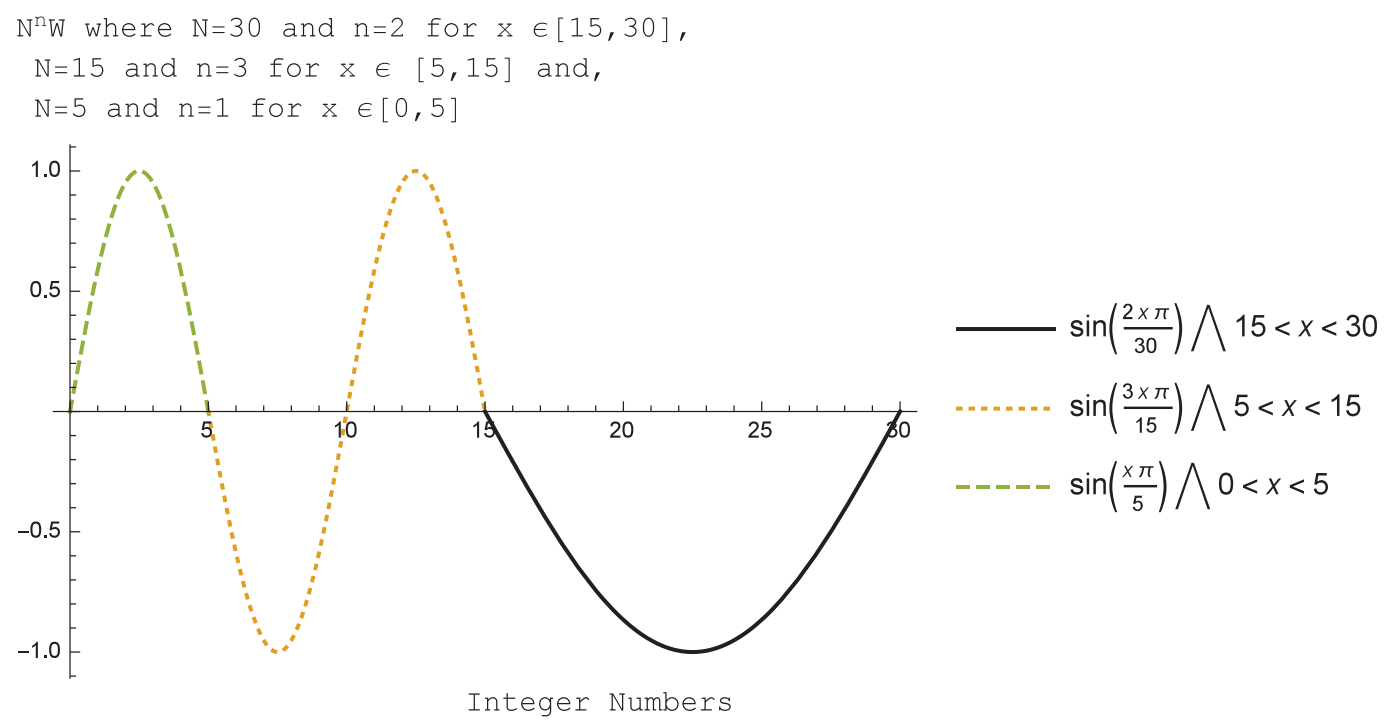

Figure 7. Factoring of number 30 in PN factors $\{2,3,5\}$. 
In fig. 7, an example of this rule of the fastest and lowest decay is shown. The $N=30$ decays by $n=2$ to $x=15$. Following, $x=15$ decays $n=3$ to $x=5$. The set of prime factors for $N=30$ is $\{2,3,5\}$.

\subsection{Factoring Prime Numbers using Prime Waves}

Graphically, the structure of the twin PNs distribution and occurrence seems to be produced by the 2 and $3 P W$. The other PWs eliminates some of these pairs by "touching" some of the numbers, undoing the pair.

These two PNs, 2 and 3, have the particularity of not being separated by any integer number. It is their $P W s$, with their initial gapless separation and their asynchronous oscillations in two different and the highest frequencies of all the $N^{n} W$, that are responsible for allowing the smallest gap between subsequent PNs.

fFig. 8 shows the $\alpha(x)$ for $N=3$, corresponding to the product of the imaginary part of the $2 P W$ and the $3 P W$. The candidate twins $(p, p+2)$ of PNs are evident in fig. $8\{(5,7),(11,13),(17,19),(23,25),(29,31)\}$. However, some of them will be undone by the $5 P W$ as, for example for the $\{(5,7)\}$ and the $\{(23,25)\}$. Other $P W \mathrm{~s}$, like the $7 P W$ or all of the others $P W \mathrm{~s}$, will undo some of the other pairs and so on for other twin PNs. Due to the use of $3 P W$, the first twin PNs $(p, p+2)$ was omitted from fig. $8\{(3,5)\}$.

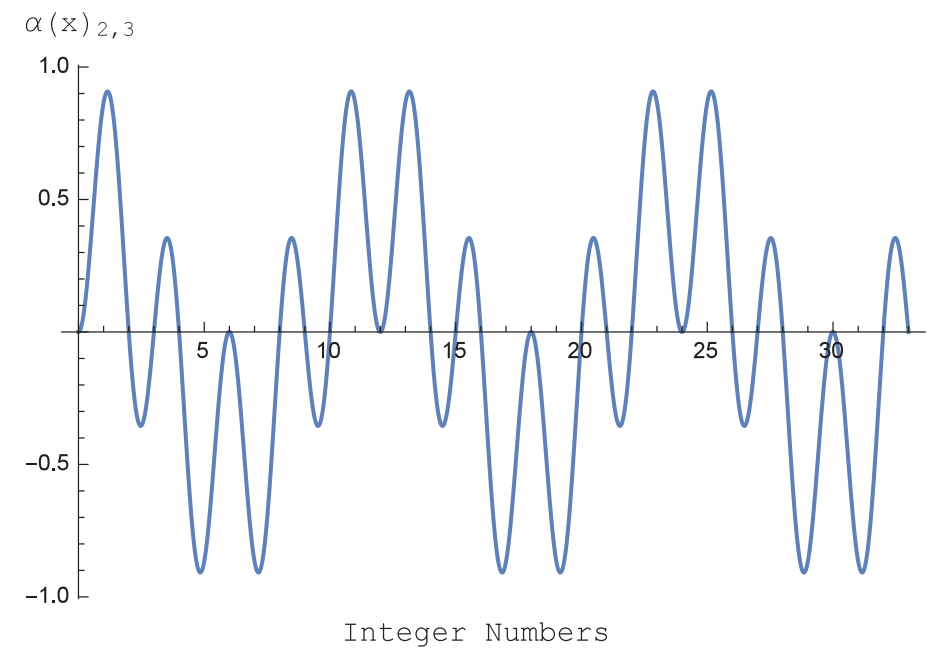

Figure 8. $\alpha(x)=\prod_{m=2}^{3} \sin \left(\frac{\pi}{m} x\right)$.

The same reasoning can be extended to another form of pairs or k-tuples of PNs.

\section{Mathematical Physics}

So far in this article it was presented theorems without any explanation or interpretation about why choose this particular treatment for the numbers. The following sections will attempt to present this explanation based on a physics approach. For this, we will consider that the numbers are the allowed energy levels for a semi-classical oscillator, following a line of thought which converts the numbers in waves in a similar way as physics converted the particles in waves with the development of quantum mechanics.

The basis of the Complex Wave model is very similar to the analysis of a particle in a one-dimensional potential box, applied to numbers. For this reason and because we are dealing with a standing wave, the model is reduced to the resolution of the time-independent Schrodinger equation.

Let us examine the typical case of a particle-wave enclosed in a potential well with the defined boundary conditions in eq. 40 , assuming that the wave is non-existent outside the potential well.

$$
V(x)=\left\{\begin{array}{l}
0 \text { for } 0<x<N \\
\infty \text { for elsewhere }
\end{array}\right.
$$

The time-independent Schrodinger equation is:

$$
\widehat{H} u_{j}=E_{j} u_{j},
$$


with the Hamiltonian (inside the box):

$$
\widehat{H}=-\frac{\hbar^{2}}{2 m} \frac{d^{2}}{d x^{2}} .
$$

The solution outside the box is:

$$
u_{j}=0
$$

and inside the box:

$$
u_{j}=e^{i k x} \wedge k \text { is } \pm \text { integer } .
$$

We need to choose linear combinations that satisfy the boundary condition that, $u_{j}(x=0)=u_{j}(x=N)=0$. We can do this easily by choosing,

$$
u_{j}=C \sin k x
$$

But since $u_{j}=0$ for $x=N$, the eigenfunctions are,

$$
u_{n}=C \sin \left(\frac{n \pi x}{N}\right)
$$

and the Schrodinger equation is,

$$
-\frac{\hbar^{2}}{2 m}\left(-\frac{n^{2} \pi^{2}}{N^{2}}\right) C \sin \left(\frac{n \pi x}{N}\right)=E C \sin \left(\frac{n \pi x}{N}\right) .
$$

There will only be a solution which satisfies the boundary conditions for a quantized set of energies.

$$
E=\frac{n^{2} \pi^{2} \hbar^{2}}{2 m N^{2}}
$$

The constant $C$ has to be normalize, for a particle in a box due to the boundary conditions that forces the totality of the wave to exist within the potential well.

$$
\left\langle u_{n} \mid u_{n}\right\rangle=\left|C^{2}\right| \int_{0}^{N} \sin \left(\frac{n \pi x}{N}\right) d x=|C|^{2} \frac{N}{2}
$$

Resulting in the following normalize equation $u_{n}$.

$$
u_{n}=\sqrt{\frac{2}{N}} \sin \left(\frac{n \pi x}{N}\right)
$$

\subsection{Complex Wave Model Transposition to the Semi Classical Oscillator}

The transposition of this line of thought from particle to numbers require certain considerations, heuristics and speculation.

Volovich proposes that major change of paradigm of physics consisted in the abandonment of the particle, waves or string as the fundamental entity in favor of considering numbers as the fundamental entity (Volovich, 2010). And using the mathematical heuristics described by Elie Zahar, where the translation of empirical content in mathematical language through physical interpretation of mathematical entities gives a new insight, the Complex Wave model translate into mathematical language the natural constraints of the distribution of PNs, interpreting the integer numbers as a manifestation of a stationary complex wave oscillating under certain conditions (Zahar, 1973). The integers will correspond to the wave materialization in the Integer set when it has only real value different from zero and imaginary value equal to zero, as if it has totally abandon other number sets and belong exclusively to the Integers set. The author believes this to be the physical interpretation of the requirement of the imaginary part of the complex wave, constituted by its sine, be zero for 
a number to be perceived as a "fully" integer. Consequently, the real and integer numbers appear as manifestations of a complex wave which implies that some numbers, interconnected by the multiplicity or divisibility relations, should belong to the same wave. If one is allowed to speculate on this, the author would say that all integers belonging to a $P W$, i.e., those which are multiple of a PN, possess a symmetry relationship with the PN on which is based the $P W$, in the sense in which a six is no more than a two rotated three times upon itself. The relationship with the p-adic universe, the congruent modulus or the consideration of multiple numbers with a relationship of "symmetry" with their least common multiple is a consequence of accepting this interpretation. This interpretation frame, seen in the light of Volovich suggestion for the continuation of a contemporary version of Einstein's programme to reduce all physics to number theory, makes more sense before their considerations on which such a reduction implies that the real numbers are realized with some probability in the Real set, and that in principle there is likely to also occur in another number set, since that it is what seems to happen in the Complex Wave model with the numbers being part real and part imaginary, simultaneously (Volovich, 2010).

The assumption of the Complex Wave model is the existence of a complex wave that, under certain boundary conditions, manifest itself in what in mathematics is referred to as "the integer numbers", as in the same way as in quantum mechanics, the wave manifest itself as "a particle". So, if we treat the complex wave the same way as quantum mechanics, or semiclassical, treats the wave that produces the particle in a semi-classical oscillator, we can understand how and why the numbers exist with certain characteristics, distribution and relationship between them. So, for the complex wave described in the Complex Wave model eq. 1, the boundary conditions only required that $u_{n}=0$ for $x=0=N$, allowing for the wave to extend to $\pm \infty$. So, $C$ can take any value we choose, since it has to be normalize to $\pm \infty$ and not to a specific interval. And it is by the mentioned argument that $x$ was chosen for the amplitude wave value, because it is the amplitude embodying the number as the number we know from the "real world" at the time that complex wave materializes in the Integer set, resulting from the equation 9. Again, as Volovich points, there are countless situations in physics that resort to the use of complex numbers to solve problems with parameters measured in the Real set. The author believes that the solution for the questions regarding PNs could be one of those situations in the field of mathematics.

The transposition of the semi-classical model of an independent harmonic oscillator time for a complex wave number generator, involve a hamiltonian operator without the need for conditions related with properties such as mass or the Planck constant or, simply, consider $m=1$ and $\hbar=1$. Then, the hamiltonian operador will have the following form, applied to the energy equation 41 :

$$
\widehat{H}=-\frac{d^{2}}{d x^{2}}
$$

The eigenfunction $u_{n}$ define as follows from the Complex wave model, since $n>2$ will only repeat energy levels:

$$
u_{n}=x \sin \left(\frac{\pi x}{N}\right)
$$

Resulting in the following allowed energy states:

$$
E_{N}(x)=-\frac{-\frac{\left(\pi^{2} x \sin \left(\frac{\pi x}{N}\right)\right)}{N^{2}}+\frac{2 \pi \cos \left(\frac{\pi x}{N}\right)}{N}}{x \sin \left(\frac{\pi x}{N}\right)},
$$

or in a more compact form,

$$
E_{N}(x)=-\frac{\csc \left(\frac{\pi x}{N}\right)\left(\frac{2 \pi \cos \left(\frac{\pi x}{N}\right)}{N}-\frac{\pi^{2} x \sin \left(\frac{\pi x}{N}\right)}{N^{2}}\right)}{x}
$$

So, if we want to find the allowed energy states available to be occupied by any given number $x=c$, all we have to do is to include all the eigenfunctions for all states before, that are already occupied by the preceding numbers such that $c>b$ and $a=2$. The energy levels for which there are a real and determined energy value solutions for eq. 53 or eq. 54 , are the energy levels available to be occupied by other integer numbers.

$$
E_{a, b}(x)=\sum_{N=a}^{b} E_{N}(x)
$$


The fig. 9 highlights the allowed energy levels coinciding with all and only PNs for $b<x \leq b^{2}$ and almost PNs and PNs for $x>b^{2}$, for which $E_{a, b}(x)$ has a solution. The not-allowed levels corresponds always to not PNs, for which the $E_{a, b}(x)$ will be of undetermined value.

Theorem 4. For all $a$, bandx integers and $b<x \leqslant b^{2}$,

$$
E_{a, b}(x)=\sum_{N=a}^{b} E_{N}(x) \neq 0 \text {, only has solution for } x \in \text { prime numbers. }
$$

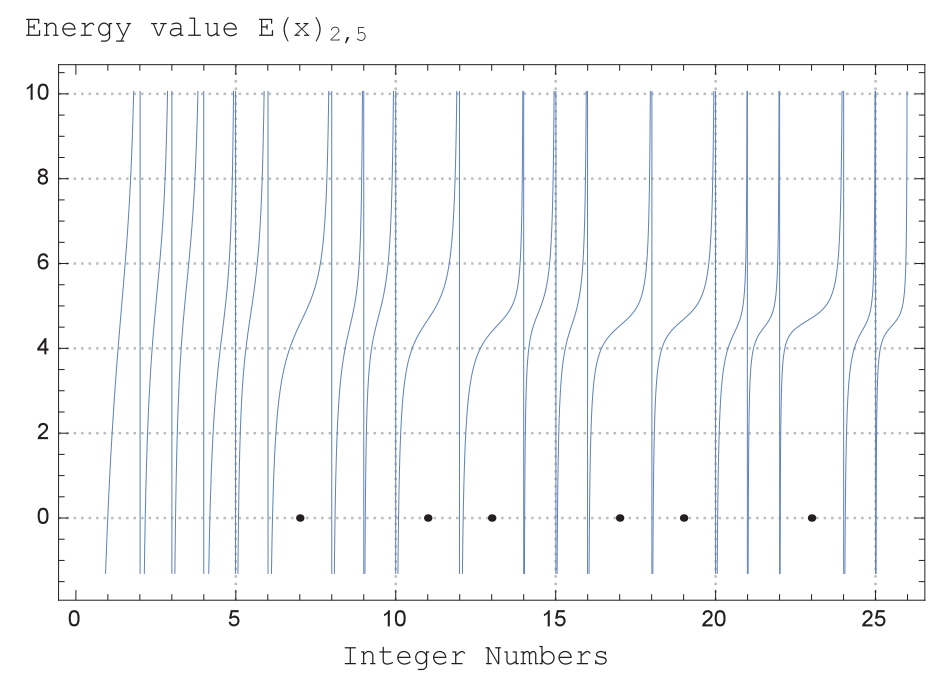

Figure 9. $E_{2,5}(x)=\sum_{N=2}^{5} E_{N}(x)$. The point marks signals all energy levels with value different from undetermined, corresponding to all PNs between 5 and 25.

Figure 10 shows the numbers and corresponding energy levels allowed, $E_{2,5}(x)$ solutions for $x$ integer, highlighting where the energy levels exists and what is its relationship with the location of PNs. Since $E_{2,5}(x)$ is constituted by eigenfunctions for $E_{2}(x), E_{3}(x)$ and $E_{5}(x)$, between 6 and 25 all energy levels correspond to PNs. From 25 to infinity, the energy levels signal all PNS and almost PNs.

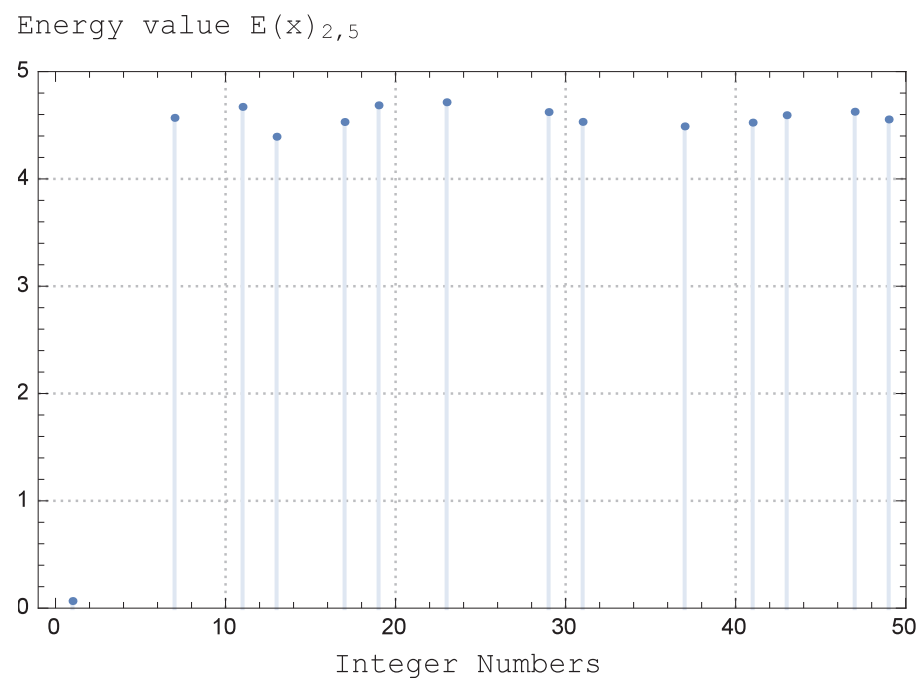

Figure 10. $E_{2,5}(x)=\sum_{N=2}^{5} E_{N}(x)$. The point marks and its height represents every energy levels with value different from undetermined and the corresponding value of that determined energy levels. From 5 to 25, all energy levels are PNs, from 25 forward are PNs and almost PNs. 


\subsubsection{Energy Spectrum for the Complex Wave Model and Riemann Zeta}

The Hilbert-Pólya conjuncture suggests a relationship between the Riemann Zeta function and the spectrum of a hermitian operator. In this work is not established the relationship between the Complex Wave model and Riemann Zeta. However, the relationship of the Riemann Zeta with PNs is well known and, in this work it is established the relationship of PNs with the spectrum of energy levels allowed in a semi-classical oscillator with hermitian operator. For this reason, the author conjecture that the relationship between the Complex Wave model, with its semi-classical harmonic oscillator interpretation, and Riemann Zeta exists. And that, the Riemann Zeta does no more than translate the spectrum defined by the time independent semi-classical harmonic oscillator, allowing the existence of PNs in certain locations.

\subsubsection{Energy Function Period}

As it was already demonstrated in the mathematical approach of the Complex Wave model, the energy function $E_{a, b}(x)$ calculated for a given $x=a$ to $b$, is periodic. Being the period calculated as, identical for the mathematical approach in eq. 19:

$$
\tau_{E_{a, b}(x)}=\prod_{N=a}^{b} N \wedge N=\{\text { all } \mathrm{PN} \text { in }[a, b]\} .
$$

The same algorithm described above, in section 2.1.3, may be used recursively to locate the energy levels corresponding to prime numbers, in a persistent database. In this approach, the $\alpha_{a, b}(x)$ is substituted by the $E_{a, b}(x)$.

\subsubsection{Number One Identified as Prime Number}

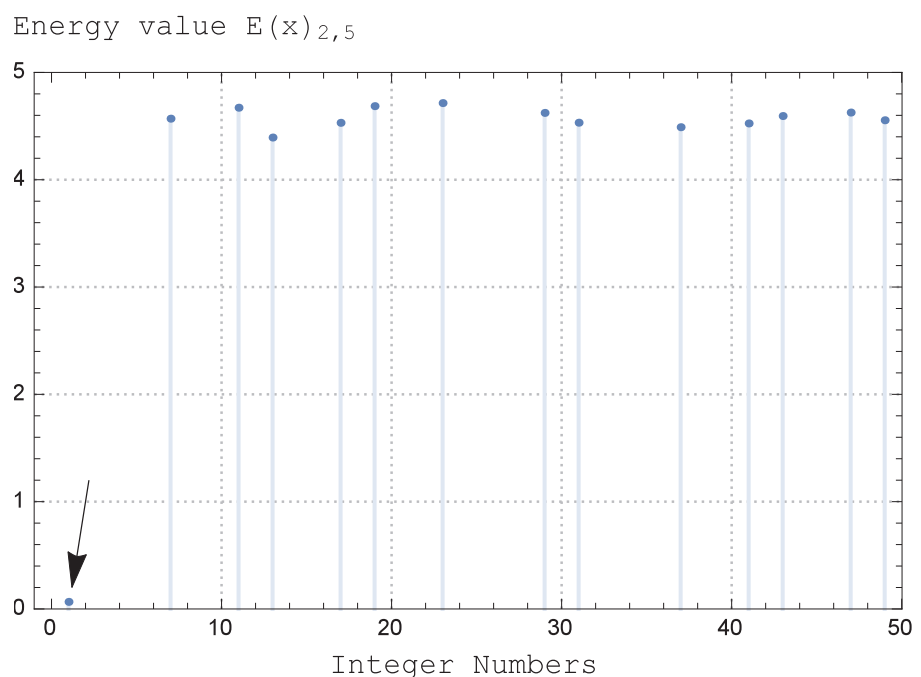

Figure 11. Energy value for $x=1$ with $E_{2,5}(x)=\sum_{N=2}^{5} E_{N}(x)$.

The number One is not considered a PN despite being divisible only by itself and by one. The main reason for so be considered is that otherwise the Fundamental Theorem of Arithmetic would be invalid. However, if we build a energy function eq. 55 to evaluate whether number One is PN, we can see that any function constructed, with $a$ and $b$ been all and any integer except one, will always have a determined and real value for $x=1$, signaling the number one as a PN. An example of these statements can be observed in fig. 11 for $E_{2,5}(x)$ with $a=2$ and $b=5$.

\subsubsection{Eigenvalues within a and $\mathrm{b}$ Boundaries}

Let us now examine the behavior of the energy function within the range of integers from $a$ to $b$. As we have seen, a function $E_{a, b}(x)$ for the eigenfunctions of $a$ and $b$, identifies PNs between $b$ and $b^{2}$. But it is also able to identify the PNs in the range $a$ to $b$. If the wave functions for all integers from $a$ to $b$ are included in the energy function, the energy levels corresponding to PNs have infinite value, while the energy levels corresponding to integers not PNs, have an indeterminate energy value. This energy function behavior is an interesting and significant capacity for creating a function with input of all integers and output of only of PNs. Since the inverse of infinite is zero and the inverse of undetermined is undetermined, 
we can represent an inverse function of the energy function with integer $x$ from $a$ to $b$, where all $E_{a, b}(x)=0$ corresponds to every $x$ PN. The fig. 12 represents all $E_{2,30}(x)$ solutions for $2<x<30$ interval and it can been seen that the set solution for $E_{2,30}(x)=0$ is exactly only and all PNs in that interval $-\{2,3,5,7,11,13,17,19,23,29\}$.

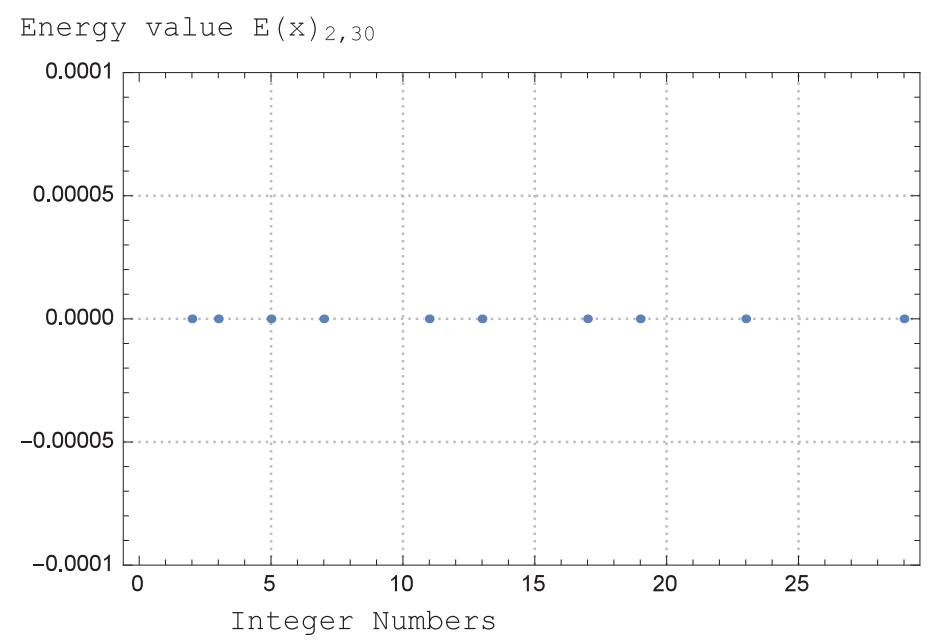

Figure 12. $\frac{1}{E_{2,30}(x)}=\frac{1}{\sum_{N=2}^{30} E_{N}(x)}$ solutions for $2<x<30$. The $\frac{1}{E_{2,30}(x)}=0$ solution set is $\{2,3,5,7,11,13,17,19,23,29\}$ corresponding to all PNs in that interval.

This behavior is extraordinary. Because, as the Riemann-Zeta, the energy function can distinguish which are numbers are PNs from within between all the numbers it contains. The same is presented in the following theorem:

Theorem 5. For all $a$, band $x$ integers and $a<x \leqslant b$,

$$
\frac{1}{E_{a, b}(x)}=\frac{1}{\sum_{N=a}^{b} E_{N}(x)}=0 \text {, only has solution for } x \in \text { prime numbers. }
$$

\subsection{Consolidating Theorems}

Theorems 4 and 5 can be consolidated in a single theorem, if we consider the inverse of the power function between a and b:

Theorem 6. For all $a, b$ and $x$ integers,

$$
\frac{1}{E_{a, b}(x)}=\frac{1}{\sum_{N=a}^{b} E_{N}(x)}\left\{\begin{array}{l}
=0, \text { only has solution for } x \in \text { prime numbers and } a<x \leqslant b \\
\neq 0, \text { only has solution for } x \in \text { prime numbers and } b<x \leqslant b^{2}
\end{array}\right.
$$

Examining the energy equation, we can see that the term responsible for identifying behavior of primes, is:

$$
z(x)=\csc \left(\frac{\pi x}{N}\right)
$$

such that:

$$
E_{N}(x)=-\frac{z(x)\left(\frac{2 \pi \cos \left(\frac{\pi x}{N}\right)}{N}-\frac{\pi^{2} x \sin \left(\frac{\pi x}{N}\right)}{N^{2}}\right)}{x} .
$$

Using $\mathrm{z}(\mathrm{x})$, we can simplify eq.59 and define $\varsigma$ function as:

$$
\varsigma_{a, b}(x)=\frac{1}{\sum_{N=a}^{b} z_{N}(x)}\left\{\begin{array}{l}
=0 \text {, only has solution for } x \in \text { prime numbers and } a<x \leqslant b \\
\neq 0 \text {, only has solution for } x \in \text { prime numbers and } b<x \leqslant b^{2} .
\end{array}\right.
$$

The figures 13 and 14 show the equation 62 solved for $\mathrm{a}=2, \mathrm{~b}=20$ and $\mathrm{x} \in] 2,50]$ and $\left.\mathrm{x} \in] 2,20^{2}\right]$, in which each dot represents a PN, if number one is considered as PN. 


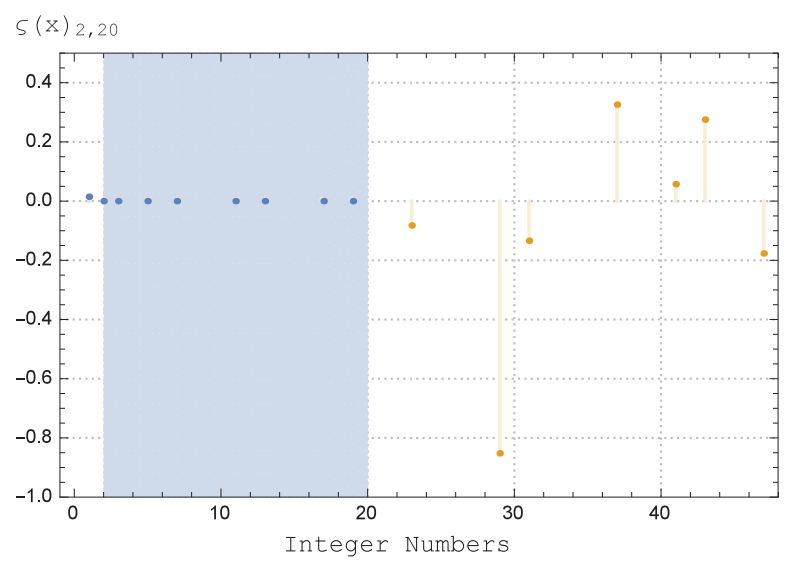

Figure 13. $\varsigma_{a, b}(x)$ solutions for $2<x<50$. The grey area represents the $\varsigma_{a, b}(x)$ solutions equals zero with $2<x \leqslant 20$ and the white area the $\varsigma_{a, b}(x)$ solutions not equal to zero and $20<x \leqslant 50$. All dots are PN, if number one is considered a PN.

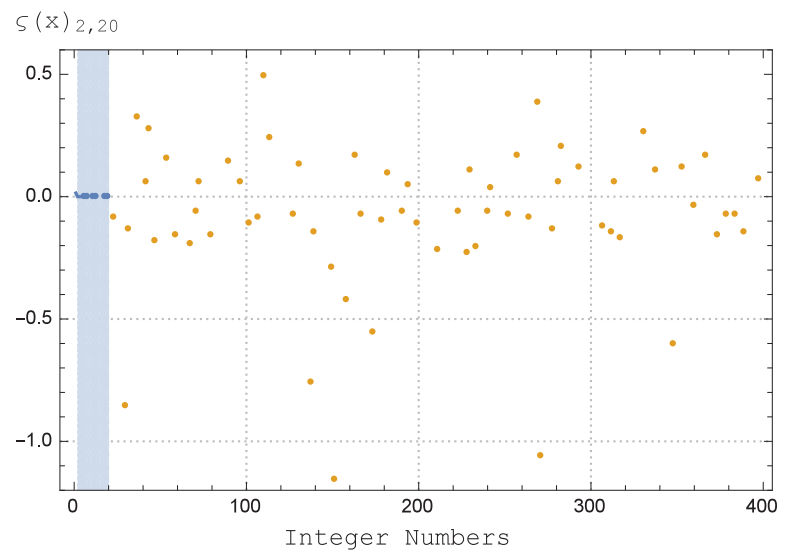

Figure 14. $\varsigma_{a, b}(x)$ solutions for $2<x<20^{2}$. The grey area represents the $\varsigma_{a, b}(x)$ solutions equals zero with $2<x \leqslant 20$ and the white area the $\varsigma_{a, b}(x)$ solutions not equal to zero and $20<x \leqslant 400$. All dots are PN, if number one is considered a PN.

\section{Conclusion}

This article shows a new way to treat integer numbers as a manifestation of a complex wave. In doing so establishes relationships between an integer number and its divisors and multiples. Thus, this approach allows to establish six theorems that determine whether an integer number is a PN, if a range of integer numbers contains PNs and where these prime numbers can occur, providing the foundation for a new formula to count PNs. Another significant result is the finding of the existence of distribution patterns for PNs that are repeated to infinity. From the computational point of view, these findings are very significant, opening the door to new ways of calculating PNs, with advantages in speed and determination of very large PNs, twins or constellations. One possibility for the use of these theorems is using electromagnetic waves interference, by creating an optical device for evaluating primality in a range of numbers, literally at the speed of light. Another possibility of using these theorems is the distribution and storage across multiple computers of the calculated patterns of PNs. It is then possible to use these stored patterns for primality evaluation of an integer, instead of having to carry out all the calculations again, thus allowing the creation of a persistent database that can be augment by reiteration. The same applies to the primality evaluation of a range of integers, and not just one at a time, with a significant increase in computational efficiency. Another important result is the ability to calculate the probability of a candidate being a PN in a certain range within a specified number of candidates. Besides the theoretical interest, in practice it can be used to set maximum likelihood in regions where such calculations are time consuming to perform, by observing regions where it is easier to perform these calculations. Also, it was demonstrated how the semi-classical interpretation of the Complex Wave model as an time independent harmonic oscillator, allows the location of the PNs by determining the energy levels allowed by the system. These results allow to respond clearly and positively to the question if the distribution of PNs is 
related to a physical system. The results of implementation of the mathematical model for the semi-classical oscillator may allow the development of new interpretations of the nature of numbers. The connection between the Riemann zeta and complex wave model was not established, but the interpretation of this model as semi-classical oscillator allows to open a new perspective on the subject.

\section{Acknowledgment}

The author would like to acknowledge to Cátia Conceição for its significant contribution in ideas, suggestions, reviews and corrections. Also, the author would like to acknowledge to Janet Sinclair for its reviewing and corrections.

\section{Endote}

$\dagger$ Another way avoiding this generalization, and perhaps more elegantly, to define this condition is to consider the following: the function of theorem 2 should be built with the first mode of oscillation for each $P W, n=1$. Therefore $\sin \left(\frac{n \pi}{m} x\right)=\sin \left(\frac{\pi}{m} x\right)$.

\$ The square of the cosine can be replaced by the $x\left\|\cos \left(\frac{n \pi}{N} x\right)\right\|$, if convenient.

\section{References}

Andrade, J. C. (2013). Hilbert-Polya conjecture, zeta functions and bosonica quantum field theories. International Journal of Modern Physics A, 28(17). http://dx.doi.org/ 10.1142/S0217751X13500723

Berry, M. V., \& Keating, J. P. (1999). The Riemann zeros and eigenvalue asymptotics. SIAM Review, 41(2), $236-266$. http://dx.doi.org/10.1137/S0036144598347497

Crandall, R., \& Pomerance, C. B. (2010). Prime Numbers: A Computational Perspective (2nd ed). New York: Springer. ISBN 9781441920508.

Dalawat, C. S. (2009). Wilson's theorem. Journal de thorie des nombres de Bordeaux, 21(3), 517-521. http://dx.doi.org/10.5802/jtnb.686

Euler, L. (1742) . De Integratione Aequationum Dierentialium altiorum graduum. pp 193-242.

Ford, K., Green, B., Konyagin, S., \& Tao, T. (2014). Large gaps between consecutive prime numbers. arXiv. http://arxiv.org/abs/1408.4505

Gutzwiller, M. C. (1990). Chaos in classical and quantum mechanics, vol 1. Springer Science \& Business Media. ISBN 0387971734.

Jaynes, E. T., \& Rosenkrantz, R. D. (1989). E.T. Jaynes: Papers on Probability, Statistics, and Statistical Physics. Springer Science \& Business Media. ISBN 9780792302131

Sierra, G. (2008). A quantum mechanical model of the Riemann zeros. New Journal of Physics, 10(3), 033016. ISSN 1367-2630. URL http://dx.doi.org/ 10.1088/1367-2630/10/3/033016

Sierra, G. (2010). A physics pathway to the Riemann hypothesis. arXiv.http://arxiv.org/abs/1012.4264

Sierra, G., \& Rodriguez-Laguna, J. (2011). H=xp Model Revisited and the Riemann Zeros. Physical Review Letters, 106(20), 200-201. http://dx.doi.org/10.1103/PhysRevLett.106.200201

Schumayer D., \& Hutchinson D. (2011). Physics of the Riemann Hypothesis. Reviews of Modern Physics, 83(2), 307-330, April 2011. ISSN 0034-6861, 1539-0756. http://dx.doi.org/doi:10.1103/RevModPhys.83.307

Volovich, I. V. (2010). Number theory as the ultimate physical theory. P-Adic Numbers, Ultrametric Analysis and Applications, 2(1), 77-87. http://link.springer.com/article/10.1134/S2070046610010061

Zahar, E. (1973). Why Did Einstein's Programme Supersede Lorentzs? (I). The British Journal for the Philosophy of Science, 24(2), 95-123. ISSN 0007-0882. http://www.jstor.org/stable/686604

Zhang, Y. (2014). Bounded gaps between primes. Annals of Mathematics, 179(3),1121-1174, May2014. ISSN0003486X. URL http://annals.math.princeton.edu/2014/179-3/p07

\section{Copyrights}

Copyright for this article is retained by the author, with first publication rights granted to the journal.

This is an open-access article distributed under the terms and conditions of the Creative Commons Attribution license (http://creativecommons.org/licenses/by/3.0/). 